\title{
Resilience of Digital Protection Relay's Power Supplies to Powerful Nanosecond Pulses
}

\author{
Vladimir Gurevich, Ph.D. \\ Central Electrical Laboratory Israel Electric Corp., Haifa, Israel
}

*Corresponding Author: Vladimir Gurevich, Central Electrical Laboratory Israel Electric Corp., Haifa, Israel.

\begin{abstract}
The article presents the results of testing the resilience of power supplies of digital protection relays to the effects of short high-voltage pulses. A strange fenomenon of the sensitivity of one of the relay types to standard nanosecond pulses5/50 ns with $1 \mathrm{kV}$ amplitude (EFT) and simultaneous resistance to more powerful standard microsecond pulses 1.2/50 $\mu$ s with amplitude $6 \mathrm{kV} \mu \mathrm{s}$ was detected. By using standard EMC filters, it is not possible to increase the stability of this digital relay to EFT.
\end{abstract}

Keywords: Electromagnetic pulse, HEMP, EFT, EMC, digital protective relays

\section{INTRODUCTION}

A Digital protection relay (DPR) is a critical kind of equipment which ensures sustainability of power systems operation. Thus, it is of utmost importance that DPRs are resilient to external electromagnetic impacts.

Electromagnetic impacts, which affect electronic equipment and impair its operation, differ in terms of frequency range, intensity and routes of entering into devices. Powerful high-voltage pulses falling into the nanoseconds range are the most dangerous type of such an impact. Transition processes, which occur during operation of high-voltage switching devices, (especially gas-insulated) may generate these pulses at power industry facilities. An electromagnetic pulse of high-altitude nuclear explosion (HEMP) [1], featuring 2.5/25 ns with the electric field's density of $50 \mathrm{kV} / \mathrm{m}$ at the ground surface, represents an even more powerful source of such an impact onto the electronic equipment of power systems.

The research of DPR resistance to electromagnetic impacts becomes more relevant in the face of a recently approved focus on intensive digitalization of the power industry, i.e. the broad use of microelectronic and microprocessor-based devices in the industry.

The most important part of a DPR is its internal power source. Thus, efficiency of other functional modules of a DPR largely depends on its integrity. On the other hand, external circuits to which it is directly connected may be a powerful source of electromagnetic impact for a DPR. This is the reason why DPR's power sources have been selected as the target of our research.

\section{ANALYSIS OF LITERATURE}

There are several publications that study sources of high-frequency electromagnetic pulses at power industry facilities and their influence on a DPR [2-6]. It should be noted that these publications discuss Japanese DPRs and the issues of the power industry in Japan. I could not find any information regarding European DPRs. Moreover, regardless of my personal extensive work experience in power industry, I did not come across any significant issues with a DPR protection during operation of highvoltage switching equipment in gas-insulated substations.

It is weird to read that the use of such simple tools such as ferrite filters on control cables and additional absorbing capacitors on input terminals of a DPR and the printed circuit board of CPU [3] solved the problem of DPR's resistance to this kind of electromagnetic impact. One can assume that a Japanese DPR does not have any electromagnetic filters and circuits which suppress noise. Another 
strange thing is that Japan adopted a new standard of test pulses for a DPR, where the amplitude of high-frequency test pulses was reduced from $4 \mathrm{kV}$ [7] to $1 \mathrm{kV}$ [8], with simultaneous modification of their shape. Thus, the standard stipulates that test pulses should be rectangular and should have a rise time of $1 \mathrm{~ns}$ and the pulse width of $100 \mathrm{~ns}(1 / 100 \mathrm{~ns})$, instead of a so-called quick nanosecond pulse featuring 5/50 ns according to [7]. Publications of Japanese authors suggest that adoption of this standard improved noise resistance of Japanese DPRs. At the same time, they do not address the issue of the source of these pulses, in other words they do not mention the necessity to build special testing equipment and use it instead of available tools for standard tests. In addition to that, there are no data about comparison tests of DPRs and their response to standard (5/50 ns) pulses and 1/100 ns pulses offered in Japan. Without these comparison tests, the use of Japanese standard with test low-amplitude (4 times lower) pulses and insignificant differences in terms of speed of rise and length is questionable. It is especially true, as in real conditions the noise signal arrives at the DPR's input terminals via rather long cables, featuring apparent inductance for nanosecond pulses and thus largely affecting their shape. Thus, the 1/100 ns pulse is unlikely to differ from 5/50 ns pulse at the DPR's input terminal.

\section{Standards and Testing Procedure}

Parameters of Electrical Fast Transient (EFT) testing procedures and technical requirements to equipment are addressed in IEC 61000-4-4:2004 [7]. Maximum amplitude of test pulses in these standards does not exceed $4 \mathrm{kV}$. However, IEC 61000-4-25 [9] suggests a two-fold higher value of amplitude for HEMP resistance testing (for typical external environment for equipment placed in enclosed concrete premises [1]). Analysis of available EFT generators [1] makes it possible to conclude that there are only two types of generators that provide the amplitude of testing pulses as stipulated by IEC 61000-4-25.

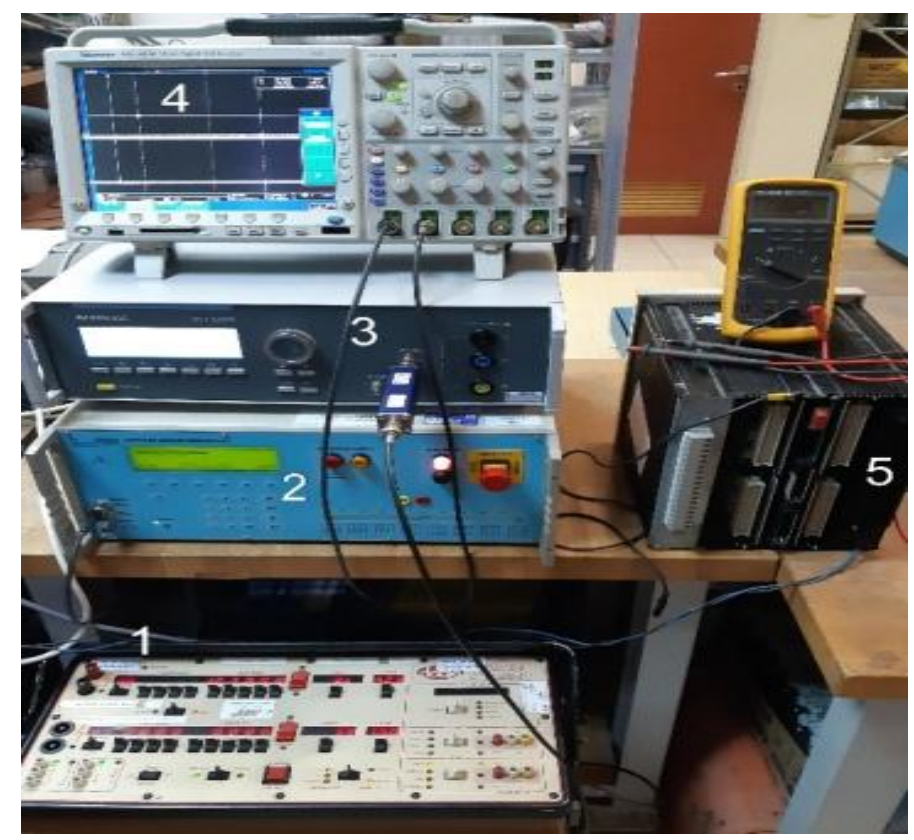

Figure1. Testing Equipment.1 - DOBLE F2253 Simulator Of Relay Protection Modes (As An External Power Source); 2 - MIG0603 Pulse Generator; 3 - 500N EFT Pulse Generator; 4 - Tektronix MSO 4034 Pulse Oscillograph; 5 - Test Object (Digital Protection Relay - DPR)

A Type 500N EFT generator manufactured by EM Test (Ametek) was used for testing purposes. This generator provides the amplitude of input pulses as high as $7 \mathrm{kV}$. In addition to that, the research used an MIG0603 generator of standard lightning pulses (1.2/50 micro-seconds) with an amplitude of up to $6 \mathrm{kV}$, (manufactured by EMC Partner) as well as a DOBLE F2253 simulator (Power System Simulator), Fig. 1.

According to IEC 61000-4-4, the standard noise represents a series of pulse bursts following each other with a certain frequency during a set period of time. Each burst consists of single short pulses following each other with a certain frequency, Fig. 2.

International Journal of Research Studies in Electrical and Electronics Engineering (IJRSEEE) Page | 39 


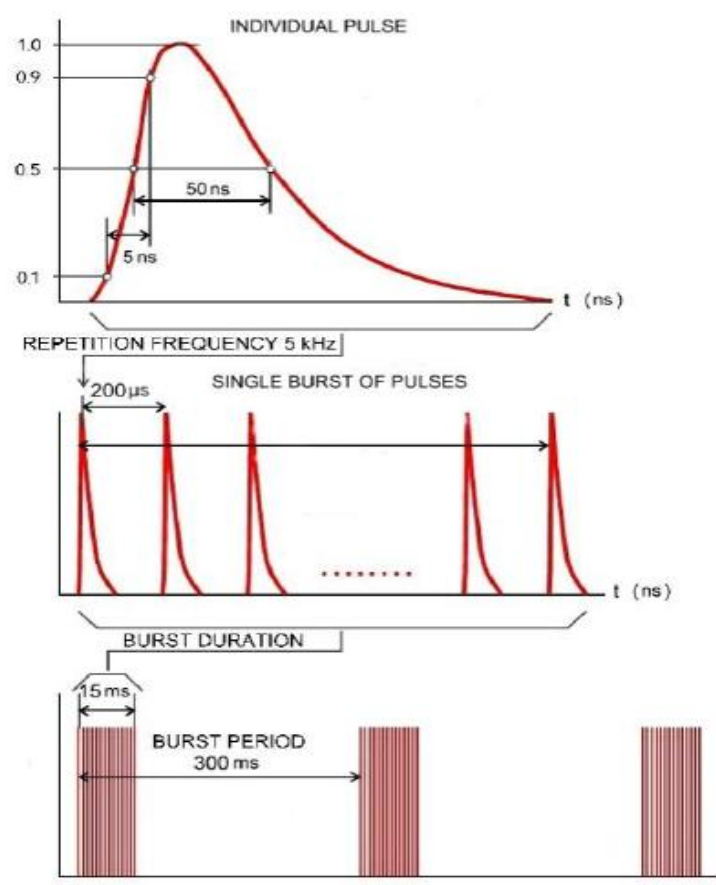

Figure2. Standard EFT Pulses (IEC 61000-4-4)
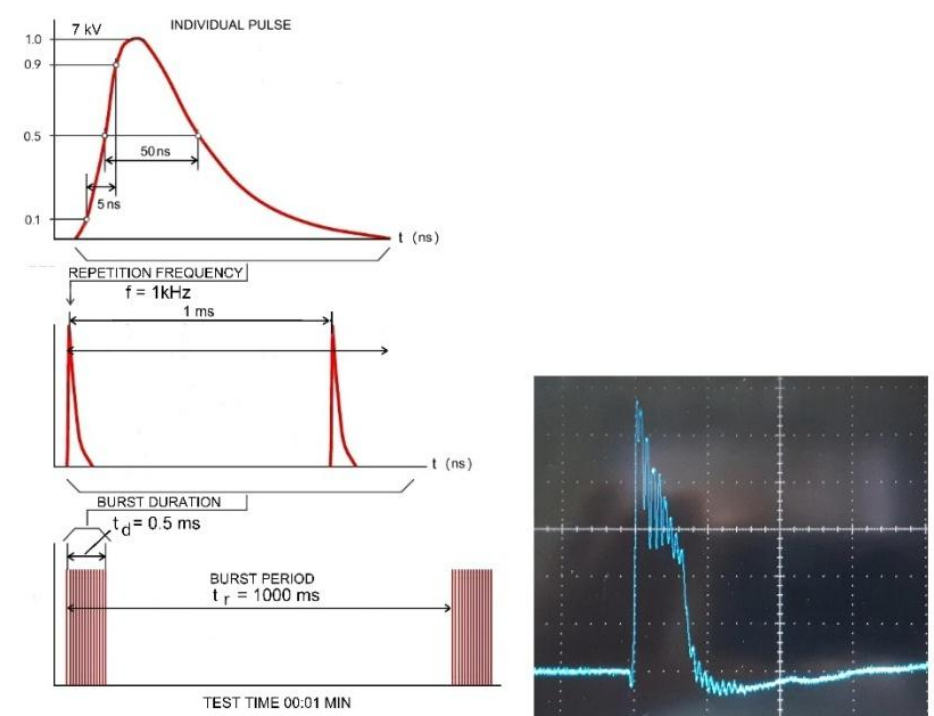

Figure3. Set-Up of the EFT Pulse Generator to Test Compliance with IEC 61000-4-25 and Oscillogram of the Real Pulse

However, IEC 61000-4-25 restricts testing of EFT resistance to applying of just a single pulse to a test object rather than pulse bursts. In other words, the standard test signal (Fig. 2) cannot be used for testing HEMP resistance. EFT generators are not meant for single pulses, but they permit to set up parameters of output pulses, and this feature was used for testing purposes. With the parameters selected, (Fig. 3) the generator sends a maximum of two separate pulses to the test object.

The following DPRs (various types, various manufacturers and various time of manufacturing) were used for testing purposes:

- SPAD330C (ABB)

- 7S5115 (Siemens)

- REC316 (ABB)

- Siprotec7SJ62 (Siemens)

- F650 (General Electric)

Each DPR was exposed to three impacts: standard burst of EFT pulses (fig. 2) with the amplitude of 1 $\mathrm{kV}$ and $4 \mathrm{kV}$, as well as single EFT pulses with the amplitude of $7 \mathrm{kV}$ (Fig. 3).

International Journal of Research Studies in Electrical and Electronics Engineering (IJRSEEE) Page | 40 
Test pulses were delivered to the supply circuits' inputs of complete switched-on DPRs receiving 230 VDC from an external power source. A special device - Coupling-Decoupling Network (CDN) - or literally, a filter built into a generator ensured protection of external power network from the test pulse impact.

The DPR's response to test pulses was recorded visually by indicators of power supply (available in any device) and by changing of status of all LED indicators available in the DPR. Changing of status of any indicator was perceived as the DPR's sensitivity to the test pulse.

\section{POWER SUPPLIES OF DPR}

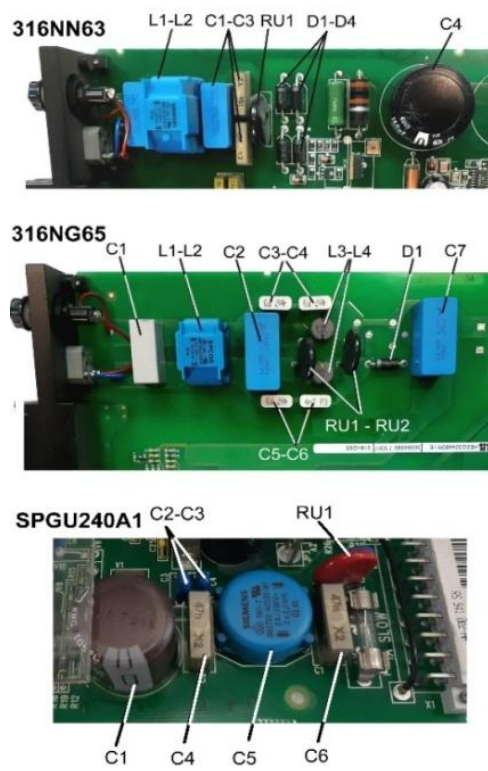

a)

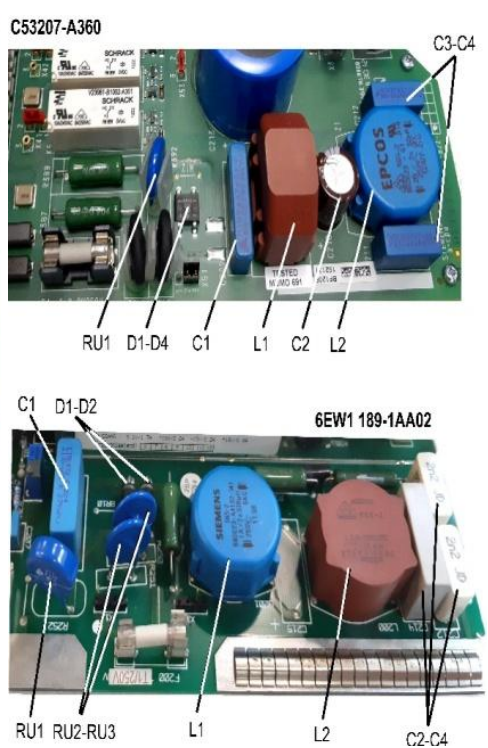

b)

Figure4. Various Types of Input Filters on DPR's Power Supplies, A) Manufactured by ABB; B) Manufactured by Siemens. In Addition to Capacitors $C$ and Chokes $L$ the Filters Also Include Varistors $R U$

Power supplies of all DPRs available in the market are of a switching type. This means that they feature a typical design, which includes an input filter, rectifier, converter of DC input voltage into high-frequency voltage, small-size step-down high-frequency transformer with a ferrite core, secondary rectifier with filters, a circuit for output voltage stabilization and adjustment (there are several circuits like this in each switching power supply). Regardless of shared mode of action, these power sources are significantly different from each other in terms of design, complexity, number of elements and size.

Since DPRs are always subject to pulse noise under a real operating environment, all power sources are compulsory equipped with input filters (Fig.4).

As a rule, these filters include high-frequency chokes $\mathrm{L}$ and capacitors $\mathrm{C}$ (designed to suppress a highfrequency noise) as well as varistors RU, which limited the noise's amplitude. Since all the power supplies contained rather complicated filters, the tests were expected to be formal and all DPRs would successfully pass them.

\section{RESUlts}

The majority of participating DPR types were really resistant to all types of test impacts. There was only one that did not pass the test. It was the REC316, manufactured by ABB with a 316NN63 type power supply. The issue was that some output voltages of this power supply and consequently some internal modules switched off at $1 \mathrm{kV}$ amplitude of a standard EFT. Later on, they automatically returned into operational conditions within several seconds upon termination of the test pulse impact. It should be noted that two LEDs on the front panel continued detecting the DPR's status change subject to the test pulse impact. This DPR showed the same response at $4 \mathrm{kV}$ amplitude of a standard EFT. Single pulses with the amplitude of $7 \mathrm{kV}$ impacting the DPR resulted in power loss in all circuits. However, several seconds later the DPR rebooted and returned into a normal state. Impact of standard pulse bursts with the increased amplitude (up to $7 \mathrm{kV}$ ) resulted in permanent damage in the power 
supply (breakdown of the key element - powerful MOSFET transistor BUZ80 type with maximal operating voltage $800 \mathrm{~V}$ and maximal impulse current 13A) and further blowing of a current-limiting resistor.

When exposing the same DPR with repaired power supply to a standard 1.2/50 $\mu$ s lightning pulse, (6 $\mathrm{kV}$ amplitude) there was no response at all, but it kept on switching off as it was affected by EFT with the amplitude of $1 \mathrm{kV}$.

Lack of detectable response of the DPR to a pulse with high amplitude and loss of efficiency (though temporarily) upon the impact of a shorter pulse with low amplitude was an unexpected finding of this experiment. An interesting fact is that previously published research (e.g. [5]) suggests that the DPR resistance is subject to amplitude of a pulse noise. Clearly, the filter does not prevent high-frequency pulses of nanosecond range from entering the internal circuits of the DPR, while providing a fair protection from pulses of microseconds range.

Thus, an attempt was made to improve the DPR's noise resistance by connecting an additional highfrequency filter ( 6 ferrite rings placed over a cable, which connects the output of a test pulse generator with a power supply) before its power supply and an additional varistor was connected parallel to the input of the power supply. The research [10] suggests that the filter performs attenuation up to $20 \mathrm{~dB}$ within $10-100 \mathrm{MHz}$ range. Unfortunately, there was no response of the DPR to test pulses.

There was another attempt to improve the DPR resistance by connecting a complex two-stage filter before the DPR's power source. This filter was very efficient in a power supply of another type and successfully passed all tests (fig. 5). The result was the same as in the first instance.

Another attempt to improve the DPR resistance stipulated the use of a special external filter. Today's market offers various types of electromagnetic filters.

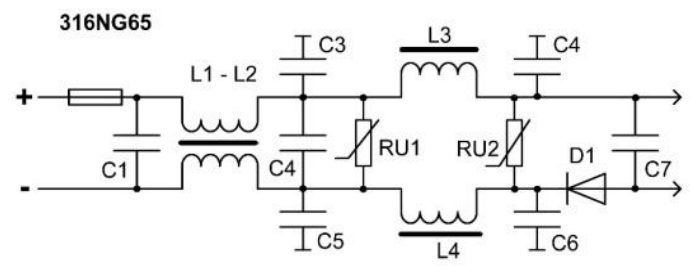

Figure5. Diagram of a Two-Stage Filter Used in 316NG65 Power Supply resilient to EFT

The most efficient of them are multi-stage LC-filters. These are manufactured in compliance with a military standard and are designed to protect equipment from HEMP. They have an attenuation level up to $100 \mathrm{~dB}$ in the frequency range from hundreds of kilohertz to dozens of Gigahertzes. These filters are manufactured by Captor, Euro-EMC, Holland Shielding Systems, MPE, ETS-Lindgren and others. They are very expensive (USD400 - 600 for a single-channel low power filter) and large in size. Multi-channel and power filters are sometimes as big as electronic equipment cabinets and their price is comparable, (Fig. 6) consequently their use in civil equipment is rather challenging.

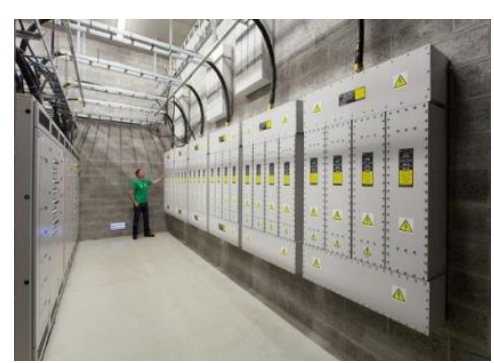

Figure6. HEMP filters manufactured by Euro-EMC (Great Britain) in a protected concrete shelter

Ordinary electromagnetic filters featuring capacitance and inductiveness (designed to protect civil equipment from electromagnetic noise) are much cheaper (USD 20 - 25) and smaller. These filters (single- and two-stage) are manufactured by dozens of companies all over the world, e.g. Shaffner, Epcos, TDK Lambda, API Technologies, Astrodyne, Qualtek Electronics and many others. The most efficient among them are two-stage filters (fig. 7).

The best samples of EMI filters from various manufacturers are not only comparable in terms of their appearance, size and price, but also in terms of almost similar attenuation level (Table 1.)

International Journal of Research Studies in Electrical and Electronics Engineering (IJRSEEE) Page | 42 
Table1. Frequency specifications of the best samples of noise-suppressing EMI filters used for civil equipment.

\begin{tabular}{|c|c|c|c|c|}
\hline \multirow{2}{*}{$\begin{array}{c}\text { Frequency, } \\
\text { MHz }\end{array}$} & \multicolumn{2}{|c|}{ Attenuation for common mode $(50 \Omega), \mathrm{dB}$} & \multicolumn{2}{c|}{ Attenuation for differential mode $(50 \Omega), \mathrm{dB}$} \\
\cline { 2 - 5 } & FN 2090-1-06 & 12-MMF-003-11-F & FN 2090-1-06 & 12-MMF-003-11-F \\
\hline 0.1 & 70 & 50 & 40 & 25 \\
\hline 1 & 75 & 90 & 80 & 65 \\
\hline 10 & 70 & 90 & 85 & 65 \\
\hline 50 & 65 & 60 & 45 & 30 \\
\hline
\end{tabular}

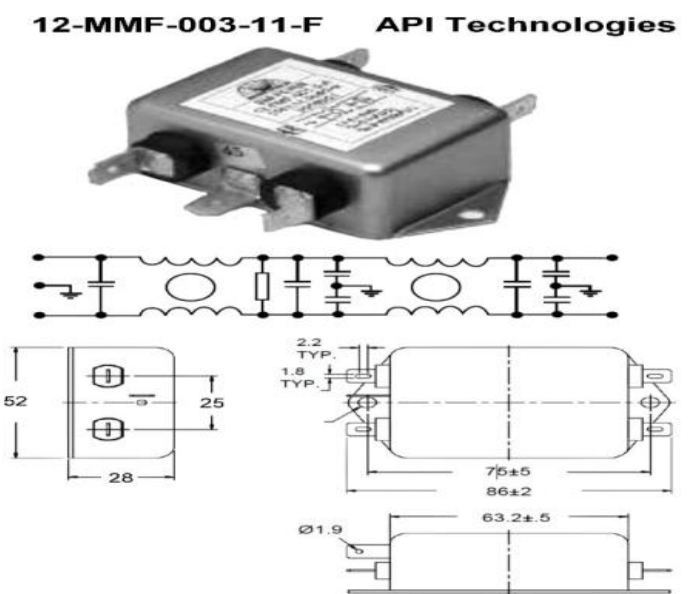

Figure7a. Two-Stage EMI Filter 12-MMF-003-11-F Manufactured by API Technologies

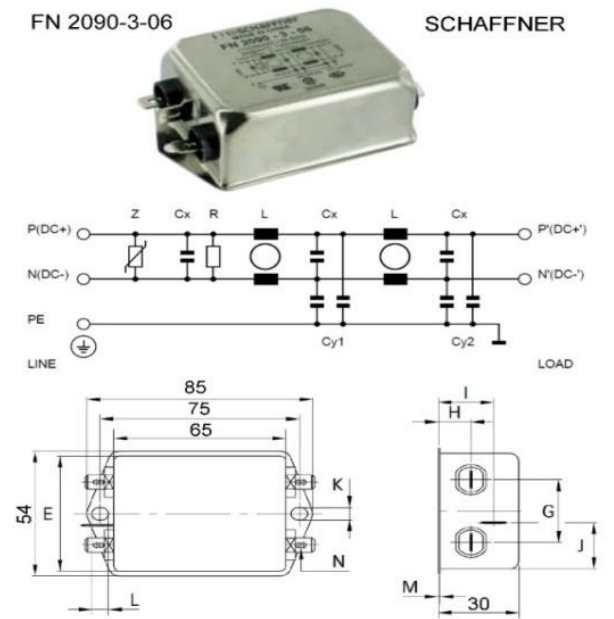

Figure7b. Two-Stage EMI Filter FN 2090-3-06 Manufactured by Schaffner

Since noise-suppressing EMI filters of this kind are not intended for high voltage, their inputs should be equipped with varistors or powerful TVS-diodes (more expensive, but faster compared to ordinary varistors).

For test purposes, an FN 2090-3-06 filter equipped with a powerful TVS-diode MAX-370 type on its input was used (Fig. 8).

Powerful TVS-diodes have recently been manufactured by two companies only: Bourns and Little fuse. Today, this list was expanded by: MDE Semiconductor, UN Semiconductor, Anova Technologies and Micro Commercial Components, which led to price reduction of these elements. For example, TVS-diodes for $430 \mathrm{~V}$ with pulse current 3 - $10 \mathrm{kA}$ cost USD $40-100$.

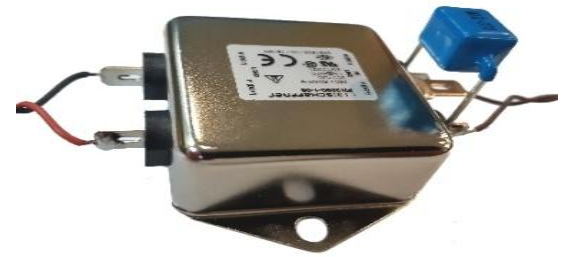

Figure8. Two-Stage Electromagnetic Filter FN 2090-3-06 Equipped With a Powerful TVS-Diode MAX-370 Type on the Input

International Journal of Research Studies in Electrical and Electronics Engineering (IJRSEEE) Page | 43 
Unfortunately, the use of a high-quality filter together with a powerful fast-responding TVS-diode was inefficient and had no effect on test findings. The reason for power supplies 316NN63 type sensitivity to a short pulse noise with restricted amplitude entering via external filters is difficult to explain.

Temporary loss of the DPR's operability during the test with its further reboot and return to the initial condition is not a permanent damage, but can be unacceptable in some cases. The fact that a manufacturer states in specifications and technical manuals (compliance with IEC 61000-4-4 and IEC 61000-4-25) does not speak for the device's responsiveness to the noise's impact. Thus, no claims can be filed to a manufacturer: after all, the device keeps on operating. However, since the response of critical equipment (e.g. DPR) to electromagnetic impact is very important, the standards have a special section, which suggests possible reaction of the device being tested, the so-called performance criteria:

a) Normal performance within limits specified by the manufacturer, requestor or purchaser;

b) Temporary loss of function or degradation of performance which ceases after the disturbance ceases, and from which the equipment under test recovers its normal performance, without operator intervention;

c) Temporary loss of function or degradation of performance, the correction of which requires operator intervention;

d) Loss of function or degradation of performance which is not recoverable, owing to damage to hardware or software, or loss of data.

In order to avoid any unexpected situations during the critical equipment's operation upon electromagnetic impact, the technical requirements of a customer should mention performance criteria in addition to a reference to the standard. On the other hand, the bidding process should make a provision for EFT-resistance testing in accordance with this standard and selected performance criterion. Putting into service should be permitted only for devices that have successfully passed the tests. Whenever the test reveals unstable operation, while even a short-term failure is not acceptable for this type of equipment, (even though it can automatically return into normal operating mode criterion "B") it should be equipped with an additional two-stage external filter. If this is also not sufficient, this equipment should not be used.

When developing HEMP protection of critical equipment, its EFT resistance needs to be confirmed in accordance with performance criteria selected. Should installation of an additional filter be necessary, it has to include a TVS-diode on its input. Alternatively, try to substitute your equipment with more resistant equipment.

\section{CONCLUSION}

1. Due to power industry digitalization, the issue of resilience of microelectronic and microprocessorbased equipment to electromagnetic impact becomes very relevant.

2. Most DPRs sustain quick pulse noise of EFT with the amplitude corresponding to requirements of the general EMC and special HEMP standards.

3. Existence of even one tested DPR type, which failed under quick pulse noise of EFT, illustrates that not all types of electronic equipment used in electric power industry are fully resilient to such noise. Thus, there is a probability that other types of DPRs or other types of microprocessor-based equipment can be subject to noise impact. So, it is recommended to test all types of critical equipment (e.g. protection relays) procured by tendering for its resilience to quick pulse noise of EFT.

4. The technical requirements of a customer should mention performance criteria in addition to a reference to the standard, while EFT-resistance testing under this standard should be conducted in accordance with the selected performance criteria.

\section{REFERENCES}

[1] Gurevich V. Protecting Electrical Equipment: Good Practice for Preventing High Altitude Electromagnetic Pulse Impacts. - De Gruyter, Berlin, 2019.

[2] Ametani A, Motoyama H., Ohkawara K., Yamakawa H., Suga N. Case Studies on Electromagnetic Disturbances of Control Circuits in Power Stations and Substations Experienced in Japan. - 42-nd International Universities Power Engineering Conference 4 - 6 September, 2007.

[3] High Frequency Switching Surge in Substation and its Effects on Operating of Digital Relays in Japan. Reference C4-304-2006, CIGRE Study Committee C4, 2006. 
[4] Matsumoto T., Kurosawa Y., Yamashita K., Tanaka T. Experience of Numerical Protective Relays Operating in an Environment with High-Frequency Switching Surge in Japan. - IEEE Transactions on Power Delivery, Vol. 21, No. 1, 2006, pp. 88 - 93.

[5] EMC within Power Plants and Substations. - Report 535 CIGRE, Working Group C4.208, 2013.

[6] Baran I., Costa M. An Analyze Coupling Mechanisms between Primary and Secondary Circuits in Air Insulated Substations. - Scientific Bulletin of the Electrical Engineering Faculty, Polytechnic University of Bucharest, 2010, No. 2 (13), pp. 7 - 12.

[7] IEC 61000-4-4:2004 Electromagnetic compatibility (EMC) - Part 4-4: Testing and measurement techniques - Electrical fast transient/burst immunity test, 2004.

[8] JEC-0103 - 2004. Standard of Low Voltage Control Circuits in Power Station and Substations, IEE Japan, 2004.

[9] IEC 61000-4-25 Ed. 1.1 b: 2012 Electromagnetic compatibility (EMC) - Part 4-25: Testing and measurement techniques - HEMP immunity test methods for equipment and systems, 2012.

[10] Gurevich V. Cheap Varistors or Expensive TVS-diodes? - International Journal of Research Studies in Electrical and Electronics Engineering (IJRSEEE), V. 4, Issue 3, 2018, pp. 1-11.

\section{AUTHOR'S BIOGRAPHY}

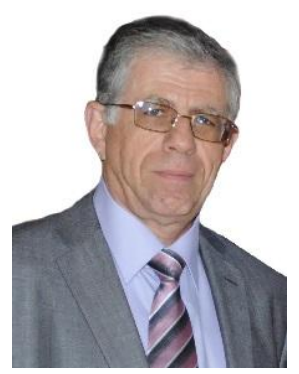

Vladimir I. Gurevich, was born in Kharkov, Ukraine, in 1956. He received an M.S.E.E. degree (1978) at the Kharkov Technical University, named after P. Vasilenko, and a Ph.D.degree (1986) at Kharkov National Polytechnic University. His employment experience includes: teacher, assistant professor and associate professor at Kharkov Technical University, and chief engineer and director of Inventor, Ltd. In 1994, he arrived in Israel and works today at Israel Electric Corp. as a senior specialist and Head of section of the Central Electric Laboratory. $\mathrm{He}$ is the author of more than 200 professional papers and 13 books and holder of nearly 120 patents in the field of electrical engineering and power electronics. In 2006 he was Honorable Professor with the Kharkov Technical University.

Citation: Vladimir Gurevich, Ph.D. "Resilience of Digital Protection Relay's Power Supplies to Powerful Nanosecond Pulses”, International Journal of Research Studies in Electrical and Electronics Engineering, 5(1), pp 38-45. DOI: http://dx.doi. org/10.20431/2454-9436.0501005

Copyright: (C) 2019 Authors. This is an open-access article distributed under the terms of the Creative Commons Attribution License, which permits unrestricted use, distribution, and reproduction in any medium, provided the original author and source are credited. 